\title{
Modelling of Deformation Resistance with Big Data and Its Application in the Prediction of Rolling Force of Thick Plate
}

\author{
Shun Hu Zhang $(\mathbb{D}$, Li Zhi Che $\mathbb{D}$, and Xin Ying Liu $(\mathbb{D}$ \\ Shagang School of Iron and Steel, Soochow University, Suzhou, China \\ Correspondence should be addressed to Shun Hu Zhang; shzhang@suda.edu.cn
}

Received 22 July 2021; Accepted 10 November 2021; Published 23 November 2021

Academic Editor: Lei Xu

Copyright (C) 2021 Shun Hu Zhang et al. This is an open access article distributed under the Creative Commons Attribution License, which permits unrestricted use, distribution, and reproduction in any medium, provided the original work is properly cited.

\begin{abstract}
The precision of traditional deformation resistance model is limited, which leads to the inaccuracy of the existing rolling force model. In this paper, the back propagation (BP) neural network model was established according to the industrial big data to accurately predict the deformation resistance. Then, a new rolling force model was established by using the BP neural network model. During the establishment of the neural network model, the data set of deformation resistance was established, which was calculated back from the actual rolling force data. Based on the data set after normalization, the BP neural network model of deformation resistance was established through the optimization of algorithm and network structure. It is shown that both the prediction accuracy of the neural network model on the training set and the test set are high, indicating that the generalization ability of the model is strong. The neural network model of the deformation resistance is compared with the theoretical one, and the maximum error is only $3.96 \%$. Furthermore, by comparison with the traditional rolling force model, it is found that the prediction accuracy of the rolling force model imbedding with the present neural network model is improved obviously. The maximum error of the present rolling force model is just $3.86 \%$. The research in this paper provides a new way to improve the prediction accuracy of rolling force model.
\end{abstract}

\section{Introduction}

Rolling force is an important parameter that is used to control the rolling process. Accurate prediction of rolling force is of great significance to optimize the processing parameter and improve the product quality and dimensional accuracy of a thick plate. Therefore, how to improve the prediction accuracy of rolling force has always been an urgent problem in this field.

For a long time, the theoretical modeling method has been considered as an ideal method to study the hot rolling process. This method can be traced back to 1925. Karman [1] established the differential equilibrium equation of force in the rolling deformation zone, which has laid a foundation for the establishment of a rolling force model. In order to obtain a more reasonable rolling model, Orowan [2] investigated the variation of yield stress and friction coefficient during rolling. Then, an analytical model of rolling force was derived by Sims [3] based on the deformation conditions proposed by Orowan and the equilibrium equation proposed by Karman. His model can reflect the functional constraint relation between rolling force and process parameters and has been widely used in practical production. In order to improve the accuracy of rolling force model, Chen et al. [4] and Li et al. [5] analyzed the friction law between the plate and roller and established a new rolling force model. Kwak et al. [6, 7] studied the influence of arm factor on rolling force and deduced a new rolling force model. Muller et al. [8] systematically considered the friction coefficients and different material parameters in the rolling process and then established a more accurate rolling force model.

Although the prediction accuracy of the above models has been continuously improved, there are still big errors since they are based on the engineering method. In order to improve the prediction accuracy of rolling force, Alexander [9] used slip-line method to analyze the hot rolling process and obtained the numerical solution of rolling force. 
Compared with the engineering method, the slip-line method can more accurately reflect the rolling deformation process, so as to realize the accurate prediction of rolling force. Collins and Dewhurst [10] presented a slip-line field covering various geometric shapes of asymmetric hot-rolled strips, which effectively increases the application range of the slip-line method. Although the slip-line method can get a more accurate solution, it is difficult to derive an analytical model of rolling force because of the difficulty in solving the nonlinear algebraic equations.

To obtain a more accurate analytical model of rolling force, some scholars have tried to analyze rolling process from the perspective of energy conservation. Zhao et al. [11] simplified the dual-stream function velocity field and established an analytical solution of the rolling force under the condition of plane deformation. On this basis, Zhang et al. [12] established a new weighted velocity field that can consider the width spread during rolling process and simplified the calculation by replacing the Mises yield criterion with the linear MY yield criterion. Finally, an analytical solution of rolling force was acquired. Considering the error between the MY yield criterion and the Mises yield criterion, some scholars have derived some other linear yield criteria, such as the equal-area yield criterion [13], angular partition yield criterion [14], and median integral yield criterion [15], to approximate the Mises yield criterion. In addition, many scholars have established different rolling velocity fields, such as the cosine velocity field [16], parabolic velocity field [17], and hyperbolic sine velocity field [18], to improve the accuracy of rolling force model. Although the above theoretical models have realized good approximation of practical rolling force, the accuracy of the existing theoretical models is still insufficient due to many inevitable assumptions.

In the process of establishing a theoretical model of rolling force, the material deformation resistance is an important parameter. Its accuracy plays an important role in improving the accuracy of the theoretical model. Johnson and Cook [19] and Khan and Huang [20], as well as Jonas et al. [21] studied the influence of external factors on deformation resistance and presented their deformation resistance model. However, just the influence of temperature, deformation degree, and deformation rate on deformation resistance are taken into account, so there is a big error. Guan et al. [22] considered the softening phenomenon in the case of great deformation degree and expressed the influence of deformation degree by a nonlinear function. Then, they derived a new deformation resistance model. Based on the deformation resistance structure proposed by Guan, Sun et al. [23], Yu et al. [24], and Liang et al. [25] have regressed many deformation resistance models for different steel grades.

In order to further improve the prediction accuracy of the deformation resistance model, Siciliano and Poliak [26] introduced the material composition as a variable into the deformation resistance model. Durrenberger et al. [27] and Molinari and Ravichandran [28] considered the effect of grain size on deformation resistance. In addition, Gao and Zhang [29], Lindgren et al. [30], and Rodriguez-Martinez et al. [31] continuously improved the accuracy of the deformation resistance model from the perspectives of dislocation, solute concentration, and vacancy. Although the introduction of these internal factors has improved the prediction accuracy of the deformation resistance model, it is difficult to measure and control the related variables in the actual production, which has limited the application of their models.

With the deepening of the research, Byon et al. [32] have also found that the deformation resistance has an important influence on the prediction accuracy of rolling force. Feng et al. [33] established a new rolling force model by combining the deformation resistance model with the Karman equation. Wang et al. [34] improved the prediction accuracy of rolling force effectively by revising the deformation resistance model with experimental data. It has been proved by the above researches that the improvement of the prediction accuracy of deformation resistance is beneficial to the improvement of the prediction accuracy of rolling force.

Based on the above research, this paper intends to use the actual production data to establish a neural network model of deformation resistance. Then, this model is used to establish a new rolling force model in order to acquire a good accuracy.

\section{Data Set and Its Preprocessing}

The purpose of the present paper is to obtain the deformation resistance data of Q345 steel from industrial big data. Because the actual production data measured by the factory is the rolling force, the deformation resistance needs to be calculated back before use. The rolling force model used in the actual hot rolling production is the Sims model, so the formula used to calculate the deformation resistance can be written as

$$
\sigma=\frac{F}{b \cdot l^{\prime} \cdot Q_{F}}
$$

where $\sigma$ is the deformation resistance of the rolled plate, $F$ is the actual rolling force, $b$ is the width of the rolled plate, $l^{\prime}$ is the length of the deformation zone, and $Q_{F}$ is the influence coefficient of the stress state. The expression of $Q_{F}$ can be written as [35]

$$
\begin{aligned}
Q_{F}= & \frac{\pi}{2} \sqrt{\frac{1-\varepsilon}{\varepsilon}} \cdot \arctan \sqrt{\frac{1-\varepsilon}{\varepsilon}}-\frac{\pi}{4}-\sqrt{\frac{R}{h}} \cdot \ln \frac{h_{r}}{h} \\
& +\frac{1}{2} \sqrt{\frac{1-\varepsilon}{\varepsilon}} \cdot \sqrt{\frac{R}{h}} \cdot \ln \frac{1}{1-\varepsilon} \\
\frac{h_{\mathrm{r}}}{h}= & 1+\frac{R}{h} \gamma^{2},
\end{aligned}
$$

where $h_{\mathrm{r}}$ is the thickness of rolled plate at the neutral plane, $\gamma$ is the neutral angle of rolled plate, and $h$ is the thickness of the plate at the exit.

Due to the complexity of (2), it is not convenient to be used online in practice, so many scholars have simplified it. In this paper, the model modified by Sun [35] can be adopted as 


$$
Q_{F}=0.8049-0.3393 \varepsilon+\left(0.2488+0.0393 \varepsilon+0.0732 \varepsilon^{2}\right) \frac{l^{\prime}}{\bar{h}},
$$

where $\varepsilon$ is the reduction rate and $\bar{h}$ is the average thickness.

According to equations (1) and (4), the formulas for calculating the actual deformation resistance according to the actual production data can be obtained through

$$
\left\{\begin{array}{l}
\sigma=\frac{F}{b \cdot l^{\prime} \cdot\left[0.8049-0.3393 \varepsilon+\left(0.2488+0.0393 \varepsilon+0.0732 \varepsilon^{2}\right) l^{\prime} / \bar{h}\right]}, \\
l \prime=\sqrt{R \prime \Delta h} \\
R_{r}^{\prime}=R_{r}\left(1+2.2 \times 10^{-5} \frac{F}{b \Delta h}\right) \\
\bar{h}=\frac{(H+h)}{2}
\end{array}\right.
$$

where $R_{r}^{\prime}$ is the flattening radius of the roller, $R_{r}$ is the initial radius of the roller, $\Delta h$ is the reduction, $\bar{h}$ is the average thickness, $H$ is the thickness at the inlet of the rolled plate, and $h$ is the thickness at the outlet of the rolled plate.

According to 1142 actual production data, the above formulas are used to calculate the actual deformation resistance of rolling plate. The data set is constructed for neural network training and verification.

In order to eliminate the influence of magnitude order difference among different parameters on the weights of neural network, the parameters in the data set are converted into dimensionless data. The transformation formula is

$$
Y=\frac{X-X_{\min }}{X_{\max }-X_{\min }},
$$

where $Y$ is the normalized data, $X$ is the input parameter, $X_{\max }$ is the maximum value of the input parameter, and $X_{\min }$ is the minimum value.

\section{Neural Network Model of Deformation Resistance Based on Big Data}

The training process of neural network includes the forward propagation of the signal and the back propagation of the error. Sigmoid function is used as the activation function for the forward propagation of the neural network, expressed as

$$
f(x)=\frac{1}{1+e^{-x}} .
$$

The mean square error (MSE) is selected as the performance evaluation function, that is as the criterion to stop the training process. Its expression is

$$
E=\frac{1}{m} \sum_{t=1}^{m}\left(\hat{y}_{i}-y_{t}\right)^{2}, \quad i=1,2, \ldots, m,
$$

where $\hat{y}_{i}$ is the training output result, $y_{i}$ is the actual output result, and $m$ is the sample number.
3.1. Selection of Training Algorithm. An excellent training algorithm can effectively improve the prediction accuracy and prevent the overfitting phenomenon. In addition to the traditional gradient descent method, many scholars have developed a variety of fast and effective training algorithms such as the Levenberg-Marquardt (LM) method, quasiNewton method, and Bayesian method. This paper compares the above three training algorithms and selects the best training algorithm. In the process of training algorithm comparison, the training parameters of the neural network are set as follows: learning rate is set to 0.01 ; validation number is set to $20 ; 70 \%$ data are selected as the training set, $15 \%$ data are as the validation set, and $15 \%$ data are as the test set.

As shown in Figure 1, with the increase of the number of hidden layer nodes, the accuracy of LM method and Bayesian method gradually improves and the accuracy of quasi-Newton method which is the lowest [36, 37]. It can be seen from Figure 1 that, as the hidden layer node increases, LM method has a large fluctuation in training, which will lead to overfitting. The Bayesian regularization method can effectively prevent the occurrence of overfitting phenomenon. Therefore, the Bayesian method can be selected as the training method of this network structure.

3.2. Determination of Network Structure. The structure of BP neural network includes input layer, hidden layer, and output layer. The nodes in the input layer and the output layer can be determined according to the actual situation.

It is known that temperature $T$, strain $\varepsilon$, and strain rate $\dot{\varepsilon}$ are the main factors that affect the material deformation resistance. Among them, strain $\varepsilon$ and strain rate $\dot{\varepsilon}$ are strongly related terms, which will cause the decrease of precision of neural network when they are taken as input variables [38]. As given, the temperature $T$, the thickness at the inlet of the rolled plate $H$, the thickness at the outlet of the rolled plate $h$, and rolling speed $v$ are selected as input variables of the neural network. So, there are four nodes in the input layer. The objective of this neural network training is to provide the deformation resistance, so there is only one network node in the output layer.

It is very important to determine the number of hidden layers and nodes of the neural network. Choosing the appropriate hidden layer structure is beneficial to obtain smaller error and better generalization ability in fewer iterations. In the selection of hidden layer structure, less layers should be selected first. So, this paper uses one hidden layer to predict the deformation resistance of materials.

In order to prevent the training results from falling into local minimum and appearing the phenomenon of overfitting, the termination error was set as 0.01. In Figure 1, when there are 10 nodes in the hidden layer, the effect of the neural network reaches a certain accuracy. Therefore, 10 nodes are selected as the initial value, and additional nodes are successively added for training until the accuracy of the neural network reaches the target requirements.

The accuracy of neural network model can be determined by network performance function and correlation 


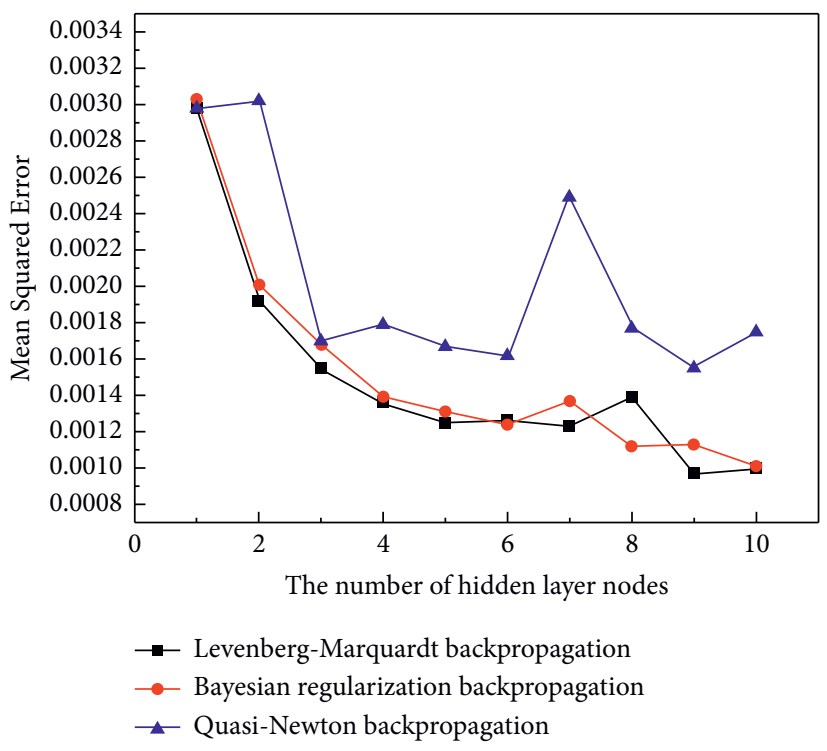

(a)

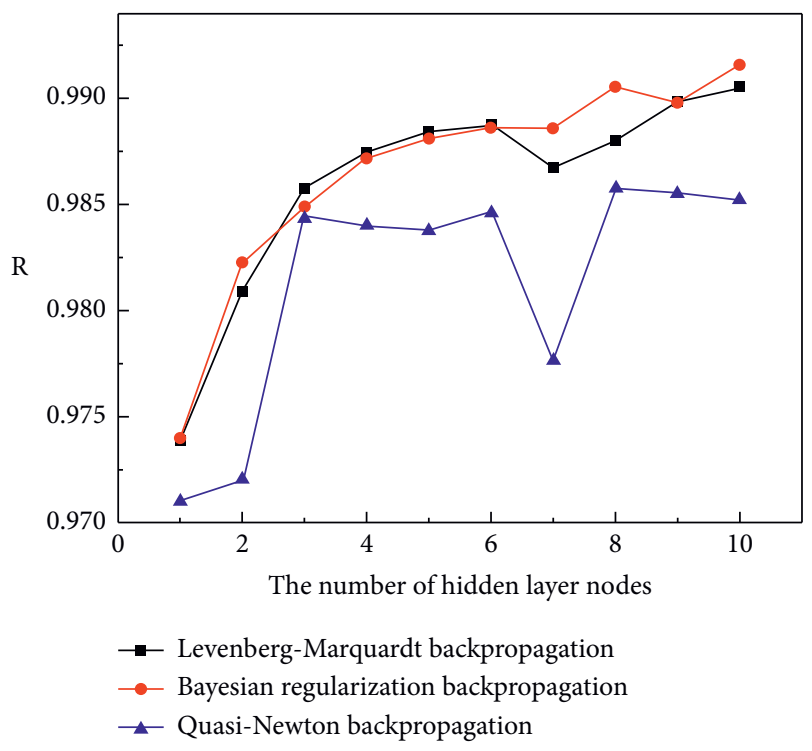

(b)

FIgURE 1: Training results: (a) MSE of different algorithms; (b) correlation coefficient $R$ of different algorithms.

coefficient $R$. The calculation formula of network performance function is shown above, while the calculation formula of correlation coefficient is

$$
R^{2}=\frac{\left(m \sum_{i=1}^{m} \hat{y}_{i} \cdot y_{i}-\sum_{i=1}^{m} \hat{y}_{i} \cdot \sum_{i=1}^{m} y_{i}\right)^{2}}{\left[m \sum_{i=1}^{m} \hat{y}_{i}^{2}-\left(\sum_{i=1}^{m} \hat{y}_{i}\right)^{2}\right]\left[m \sum_{i=1}^{m} y_{i}^{2}-\left(\sum_{i=1}^{m} y_{i}\right)^{2}\right]}
$$

For the neural network model, the smaller the performance function is and the closer the correlation coefficient is to 1 , the closer the result is good.

As shown in Figure 2, when the number of nodes increases to 13 , the performance test of the neural network meets the requirement of MSE, but at this time, the regression coefficient $R$ value of the neural network is lower than that with the other nodes. When the number of hidden layer nodes increases to 15 , the neural network not only meets the performance test requirements but also has the best regression for training data. Table 1 shows the MSE of BP neural network with different number of nodes. The results show that when the number of hidden nodes is 15 , the calculation accuracy is the highest.

In summary, the structure of the neural network is finally determined to be 4-15-1. The BP neural network structure model is shown in Figure 3.

3.3. Performance Evaluation. The final results of mean square error, error distribution, and correlation coefficient $R$ are shown in Figure $4-6$.

Figure 4 illustrates the variation of mean square error with epoch. At the beginning of the training, the model error drops rapidly and then fluctuates in the later period. It can be seen from Figure 4 that the neural network model is terminated when the network iteration is 673 times. Its MSE is 0.0008564 .
Figure 5 illustrates the error distribution on different data sets. In Figure 5, 80\% of the sample errors of the neural network model obtained in this study are within $5 \%$, and $94 \%$ of the sample errors are within $10 \%$. The error distributions of training and test data concentrate near 0 . This result shows that this network model has good prediction accuracy.

Figure 6 shows the prediction results upon different data sets. The $R$ value of each part is about 0.99 . It means that the data obtained by the neural network has a high approximation to the actual data.

\section{Comparison and Verification}

4.1. Comparison of Deformation Resistance. The traditional regression model of deformation resistance for Q345 can be written as [15]

$$
\sigma_{s}=3583.15 e^{-2.233 \times 10^{-3} T} \cdot \varepsilon^{0.42437} \cdot \dot{\varepsilon}^{-0.3486 \times 10^{-3} T+0.46339},
$$

where $\varepsilon$ is the equivalent strain; $\dot{\varepsilon}$ is the equivalent strain rate; and $T$ is the Kelvin temperature.

Figure 7 shows the deformation resistance of each pass in the rolling process. Table 2 shows the theoretical error and the $\mathrm{BP}$ error of deformation resistance for each pass. It can be seen from Table 2 that the deformation resistance error obtained by the traditional regression model fluctuates greatly. The errors in the first 4 passes of the initial rolling and in the last 5 passes of rolling are too big, exceeding the engineering allowable error range of $15 \%$. In the first 4 passes, the strain is less than 0.1. At this situation, the deformation resistance increases rapidly with the increase of strain. That is to say, a small change of strain will lead to a severe fluctuation of the deformation resistance, which will lead to the inaccurate prediction of deformation resistance. In the last 5 passes, the strain rates are greater than $10 \mathrm{~s}-1$. For 


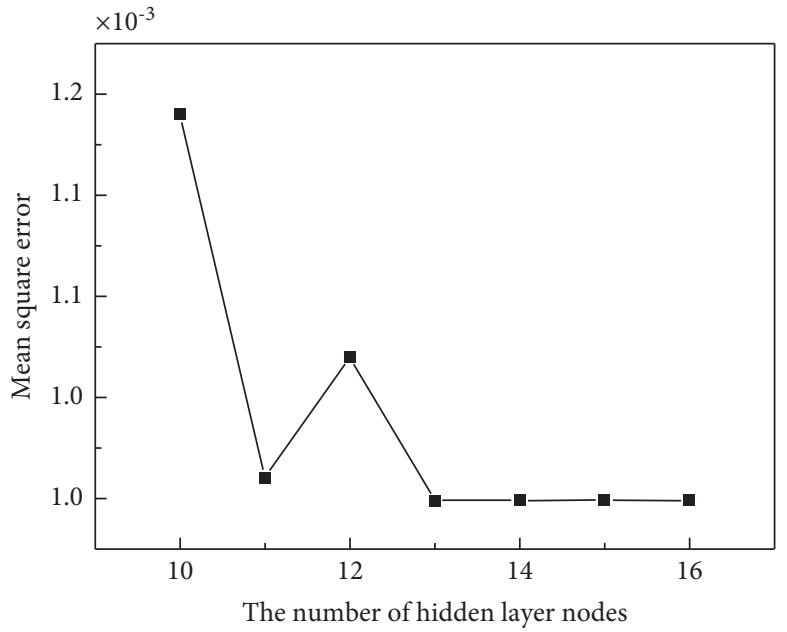

(a)

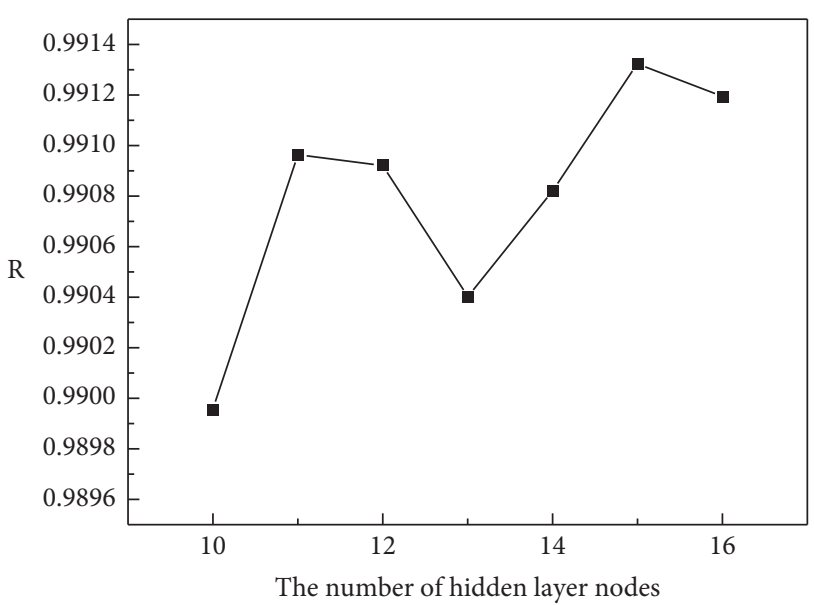

(b)

FIGURE 2: Training results: (a) the MSE of BP network with different number of nodes; (b) correlation coefficient of BP network with different number of nodes.

TABLE 1: Comparison of training accuracy with different number of nodes.

\begin{tabular}{lcc}
\hline The number of hidden layer nodes & Minimum accuracy (\%) & Average accuracy (\%) \\
\hline 13 & 94.35 & 97.48 \\
14 & 93.38 & 97.73 \\
15 & 95.57 & 98.15 \\
16 & 93.93 & 97.64 \\
\hline
\end{tabular}

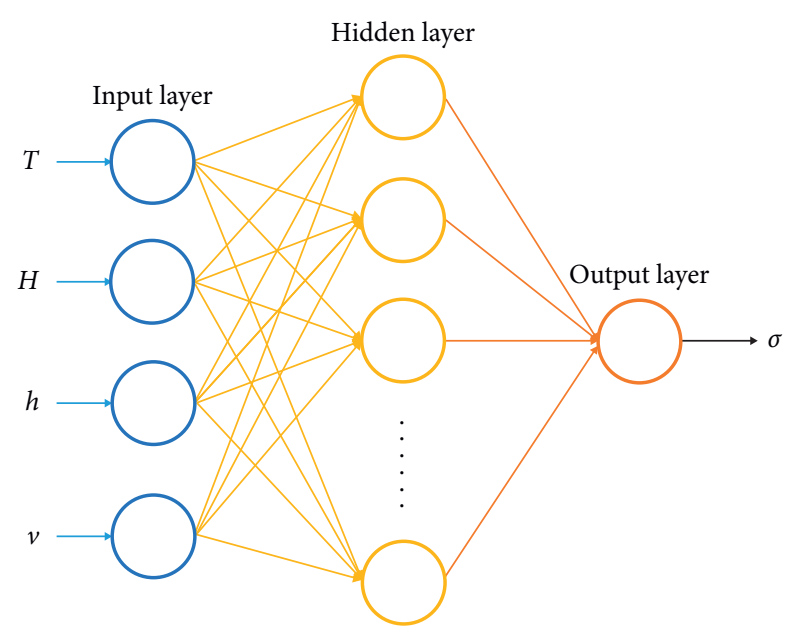

FIGURE 3: BP neural network structure model.

this case, big errors will arise since the effect of work hardening at a high strain rate has not been considered in the traditional regression model proposed by SAITO [39]. Compared with the traditional regression model, the minimum error of deformation resistance by the $\mathrm{BP}$ neural network is $0.26 \%$, and the maximum error is $3.96 \%$. The BP neural network can provide a stable and high precision. The primary reason for this good result is that the BP neural network is built on the production data which can reflect the real parameter relationship effectively. Moreover, the BP neural network has a strong nonlinear mapping ability of input parameters and output parameters.
4.2. Comparison of Rolling Force. The present rolling force can be obtained by replacing the traditional regression model of deformation resistance by the present BP neural network model. So, we can have

$$
F=\sigma_{B P} \cdot b \cdot l l \cdot Q_{F},
$$

where $\sigma_{\mathrm{BP}}$ is the deformation resistance model predicted by BP neural network.

The comparison result of the rolling force is shown in Figure 8. The rolling force calculated by traditional 


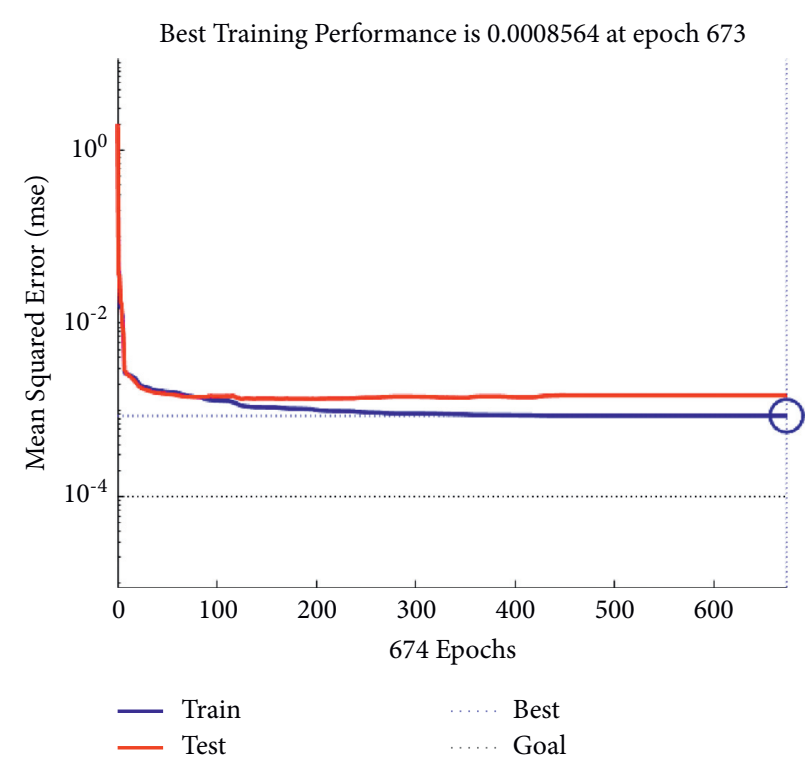

FIgURE 4: Variation of mean square error during training.

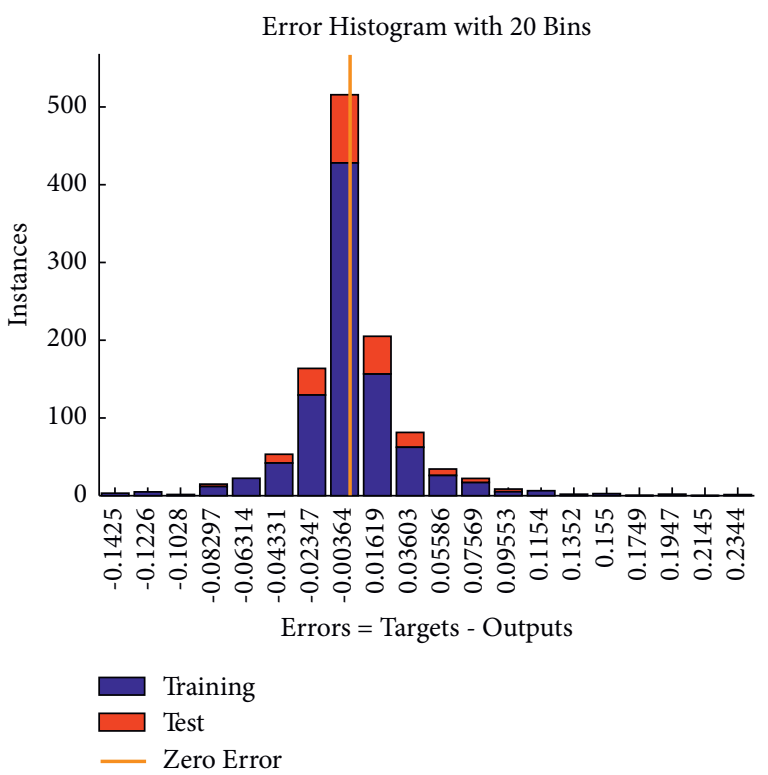

Figure 5: Histogram of error distribution.

regression model is greatly different from the actual data, while the rolling force model with big data is consistent with the actual data. The prediction errors of rolling force for each pass are shown in Table 3. The results show that the minimum error of the traditional regression model is 3.48\%, while the minimum error of the rolling force model with big data is $0.27 \%$ and the maximum error is $3.86 \%$. The rolling force model in this paper is far better than the traditional regression model.
It is known that the stress state coefficient and deformation resistance are the two factors that affect the calculation accuracy of rolling force, of which the stress state coefficient has been checked to be accurate [35, 40]. Therefore, the precision of the rolling force will depend on the precision of deformation resistance. This can explain the reason why the prediction accuracy of rolling force increases with the increase of the prediction accuracy of deformation resistance. 

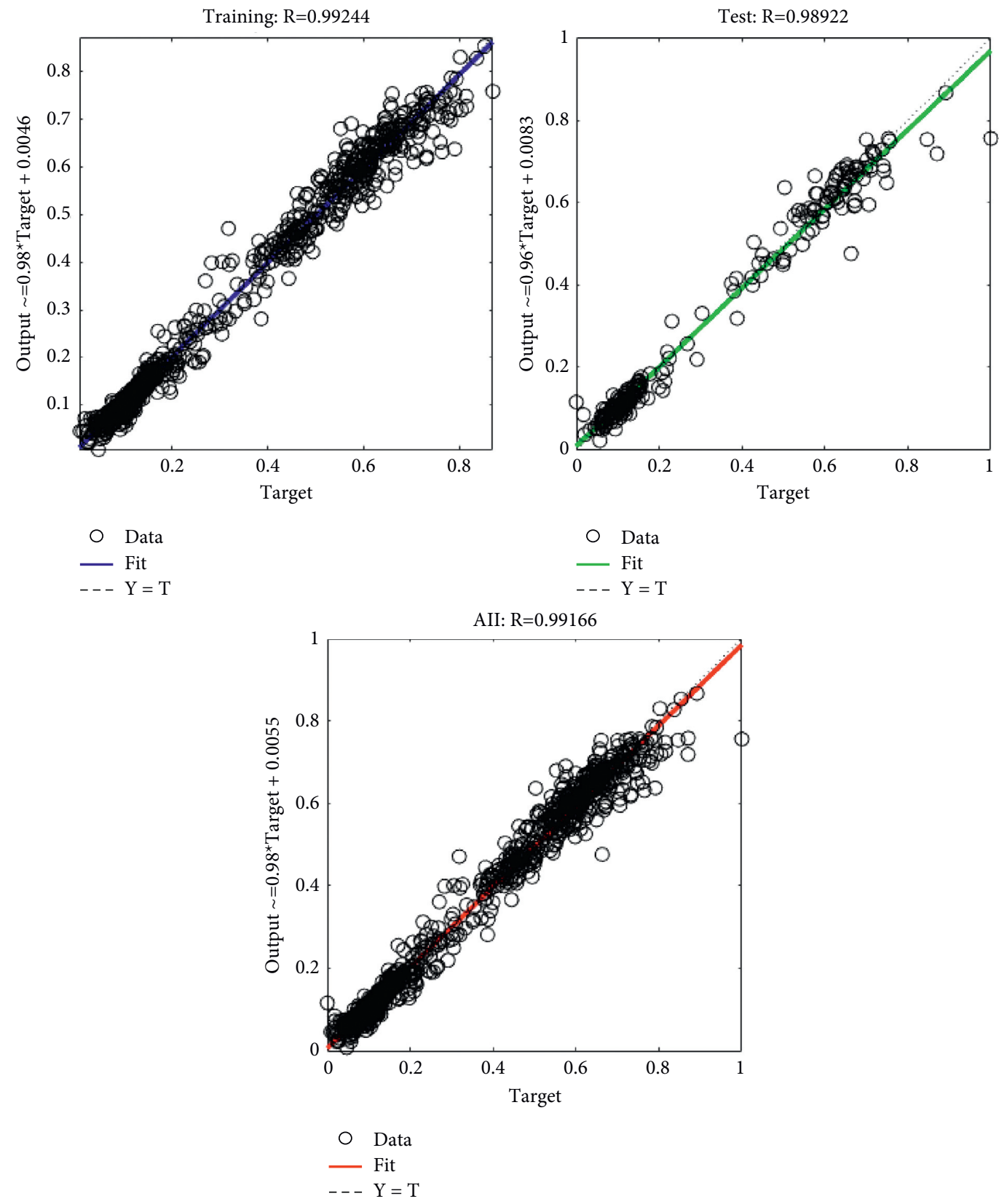

Figure 6: Prediction results upon different data sets. 


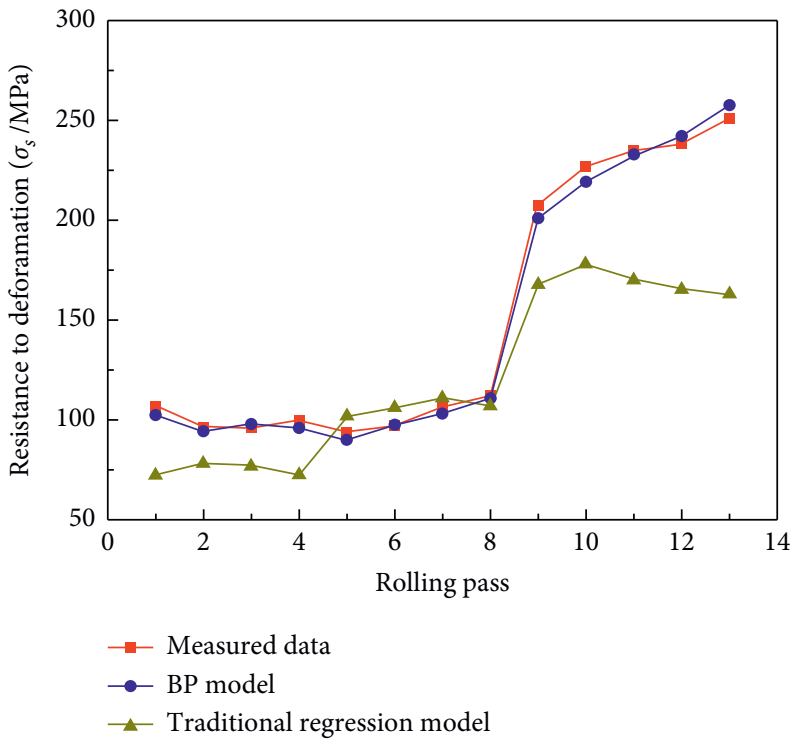

FIGURE 7: Comparison of deformation resistance.

TABLE 2: The theoretical error and the BP error of deformation resistance for each pass.

\begin{tabular}{lccccc}
\hline Rolling pass & Theoretical error (\%) & BP error (\%) & Rolling pass & Theoretical error (\%) & BP error (\%) \\
\hline 1 & $-29.58 \%$ & $3.96 \%$ & 8 & -3.56 & -16.60 \\
2 & $-17.08 \%$ & $2.05 \%$ & 9 & -18.75 & 3.22 \\
3 & $-21.70 \%$ & $-1.83 \%$ & 10 & -26.99 & 3.49 \\
4 & $-24.26 \%$ & $3.74 \%$ & 11 & -31.80 & -1.83 \\
5 & $12.93 \%$ & $3.94 \%$ & 12 & -36.79 & -2.66 \\
6 & $8.90 \%$ & $-0.26 \%$ & 13 & & \\
7 & $7.40 \%$ & $3.62 \%$ & & & \\
\hline
\end{tabular}

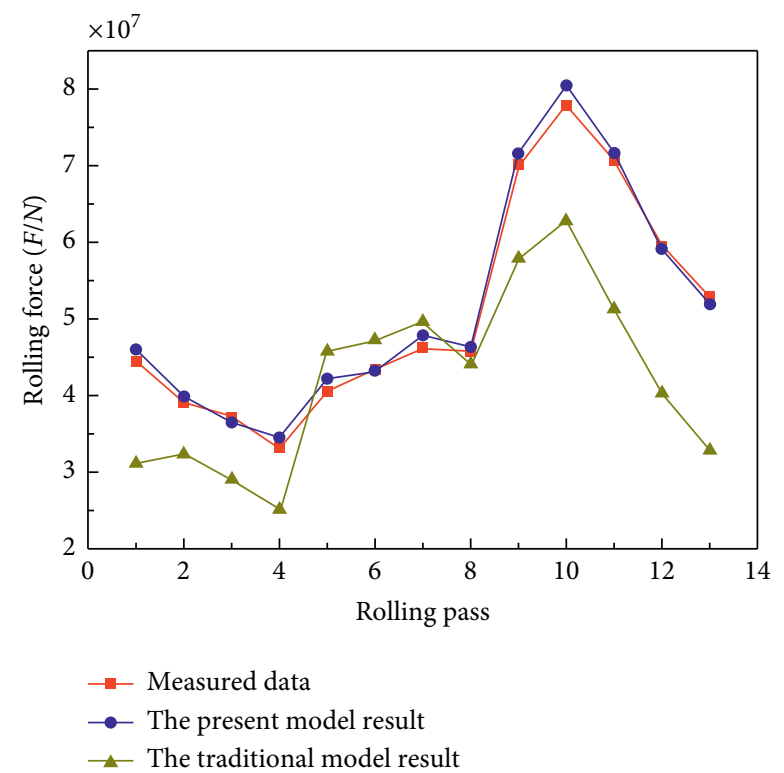

FIgURE 8: Comparison of rolling force. 
TABLE 3: The theoretical error and the model error of deformation resistance for each pass.

\begin{tabular}{lccccc}
\hline Rolling pass & Theoretical error (\%) & Model error (\%) & Rolling pass & Theoretical error (\%) & Model error (\%) \\
\hline 1 & -29.97 & $3.38 \%$ & 8 & $-3.48 \%$ & $-17.37 \%$ \\
2 & -17.17 & $2.15 \%$ & 9 & $-19.43 \%$ & $2.16 \%$ \\
3 & -21.76 & $-1.79 \%$ & 10 & $-27.35 \%$ & $3.22 \%$ \\
4 & -24.32 & $3.81 \%$ & 11 & $-32.33 \%$ & $-0.49 \%$ \\
5 & 12.71 & $3.86 \%$ & 12 & $-37.87 \%$ & $-1.83 \%$ \\
6 & 8.94 & $-0.27 \%$ & & & \\
7
\end{tabular}

\section{Conclusion}

(1) According to the actual production data, the BP neural network model is established for the deformation resistance of a thick plate. The network structure is determined as 4-15-1. By comparing different training algorithms, the Bayesian method has been chosen since it occupies the advantages of higher accuracy and less tendency of overfitting.

(2) According to the established BP neural network model, this paper has realized the accurate and stable prediction of deformation resistance in the rolling process of thick plates. In the verified 13 passes, the maximum error of the neural network prediction results is $3.96 \%$, which is far lower than the maximum error of the theoretical regression model. The neural network model is suitable for actual production conditions and can provide better guidance for actual production.

(3) In the 13 passes, the maximum prediction error of the model in this paper is $3.86 \%$, which has realized the stable and accurate output of rolling force. Moreover, it is found that the prediction accuracy of rolling force increases with the increase of deformation resistance accuracy.

\section{Data Availability}

The data set used to support the findings of this study are available from the corresponding author upon request.

\section{Conflicts of Interest}

The authors declare that they have no conflicts of interest.

\section{Acknowledgments}

This research was supported by the National Natural Science Foundation of China (Grant nos. U1960105 and 52074187), the Outstanding Youth Foundation of Jiangsu Province (Grant no. BK20180095), and the National Under Graduate Innovation and Entrepreneurship Training Program (202010285033Z).

\section{References}

[1] T. v. Kármán, "8. Beitrag zur Theorie des Walzvorganges," ZAMM - Journal of Applied Mathematics and Mechanics/ Zeitschrift für Angewandte Mathematik und Mechanik, vol. 5, no. 2, pp. 139-141, 1925.
[2] E. Orowan, "The calculation of roll pressure in hot and cold flat rolling," Proceedings - Institution of Mechanical Engineers, vol. 150 , no. 1 , pp. 140-167, 1943.

[3] R. B. Sims, "The calculation of roll force and torque in hot rolling mills," Proceedings - Institution of Mechanical Engineers, vol. 168, no. 1, pp. 191-200, 1954.

[4] S. Chen, W. Li, and X. Liu, "Calculation of rolling pressure distribution and force based on improved Karman equation for hot strip mill," International Journal of Mechanical Sciences, vol. 89, pp. 256-263, 2014.

[5] W.-G. Li, C. Liu, B. Liu, B.-k. Yan, and X.-H. Liu, "Modeling friction coefficient for roll force calculation during hot strip rolling," International Journal of Advanced Manufacturing Technology, vol. 92, no. 1-4, pp. 597-604, 2017.

[6] W. J. Kwak, Y. H. Kim, H. D. Park, J. H. Lee, and S. M. Hwang, "FE-based on-line model for the prediction of roll force and roll power in hot strip rolling," ISIJ International, vol. 40, no. 10, pp. 1013-1018, 2000.

[7] W. J. Kwak, J. H. Lee, S. M. Hwang, and Y. H. Kim, "A precision on-line model for the prediction of roll force and roll power in hot-strip rolling," Metallurgical and Materials Transactions A, vol. 33, no. 10, pp. 3255-3272, 2002.

[8] M. Müller, A. Steinboeck, K. Prinz, and A. Kugi, "Optimal parameter identification for a hydrodynamic roll gap model in hot strip rolling," IFAC-PapersOnLine, vol. 51, no. 21, pp. 195-200, 2018.

[9] J. M. Alexander, "A slip line field for the hot rolling process," Proceedings - Institution of Mechanical Engineers, vol. 169, no. 1, pp. 1021-1030, 1955.

[10] I. F. Collins and P. Dewhurst, "A slipline field analysis of asymmetrical hot rolling," International Journal of Mechanical Sciences, vol. 17, no. 10, pp. 643-651, 1975.

[11] D.-w. Zhao, S.-h. Zhang, C.-m. Li, H.-y. Song, and G.-d. Wang, "Rolling with simplified stream function velocity and strain rate vector inner product," Journal of Iron and Steel Research International, vol. 19, no. 3, pp. 20-24, 2012.

[12] S. H. Zhang, X. D. Chen, J. X. Hou, and D. W. Zhao, “Analysis of broadside rolling for heavy plate by weighted velocity field and mean yield criterion," Meccanica, vol. 51, no. 5, pp. 1189-1199, 2016.

[13] J.-z. Cao, D.-w. Zhao, S.-h. Zhang, W. Peng, S.-z. Chen, and D.-h. Zhang, "Analysis of hot tandem rolling force with logarithmic velocity field and EA yield criterion," Journal of Iron and Steel Research International, vol. 21, no. 3, pp. 295-299, 2014.

[14] S. H. Zhang, X. N. Wang, D. W. Zhao et al., "Analysis of broadside rolling force for hot heavy plate using angle bisector yield criterion," Journal of University of Science and Technology Liaoning, vol. 39, no. 06, pp. 418-423, 2016.

[15] X. R. Jiang, S. H. Zhang, and C. J. Wang, "Analysis of elliptical velocity field in heavy plate rolling by integral mean value yield criterion," Journal of Harbin Institute of Technology, vol. 52, no. 05, pp. 41-48, 2020. 
[16] D.-H. Zhang, Y.-M. Liu, J. Sun, and D.-W. Zhao, "A novel analytical approach to predict rolling force in hot strip finish rolling based on cosine velocity field and equal area criterion," International Journal of Advanced Manufacturing Technology, vol. 84, no. 5-8, pp. 843-850, 2016.

[17] W. Peng, D. Zhang, and D. Zhao, "Application of parabolic velocity field for the deformation analysis in hot tandem rolling," International Journal of Advanced Manufacturing Technology, vol. 91, no. 5-8, pp. 2233-2243, 2017.

[18] J. Sun, Y.-M. Liu, Y.-K. Hu, Q.-L. Wang, D.-H. Zhang, and D.-W. Zhao, "Application of hyperbolic sine velocity field for the analysis of tandem cold rolling," International Journal of Mechanical Sciences, vol. 108-109, pp. 166-173, 2016.

[19] G. R. Johnson, W. H. Cook, R. Johnson et al., "A constitutive model and data for materials subjected to large strains, high strain rates, and high temperatures," in Proceedings of the 7th International Symposium of Ballistics, pp. 541-547, Hague, The Netherlands, April 1983.

[20] A. S. Khan and S. Huang, "Experimental and theoretical study of mechanical behavior of 1100 aluminum in the strain rate range 10-5-104s-1," International Journal of Plasticity, vol. 8, no. 4, pp. 397-424, 1992.

[21] J. J. Jonas, C. M. Sellars, and W. J. M. Tegart, "Strength and structure under hot-working conditions," Metallurgical Reviews, vol. 14, no. 1, pp. 1-24, 1969.

[22] K. Z. Guan, J. H. Zhou, and Q. S. Zhu, "An experimental study of the resistance to plastic deformation of hot rolling metals," Journal of Beijing University of Iron and Steel Technology, vol. 01, pp. 123-139, 1983.

[23] J.-q. Sun, H. Dai, and Y.-c. Zhang, "Research on mathematical model of thermal deformation resistance of X80 pipeline steel," Materials \& Design, vol. 32, no. 3, pp. 1612-1616, 2011.

[24] C. S. Yu, Z. W. Zheng, M. L. Wang, and G. T. Zhang, "A study on hot-rolling deformation resistance of A TRIP steel," Applied Mechanics and Materials, vol. 692, pp. 359-365, 2014.

[25] J. L. Liang, Y. L. Feng, J. Z. Yin, D. Q. Cang, and H. Li, “The mathematic model of deformation resistance of S50C medium carbon steel in hot rolling process," Advanced Materials Research, vol. 652-654, pp. 652-2047, 2013.

[26] F. Siciliano and E. I. Poliak, "Modeling of the resistance to hot deformation and the effects of microalloying in high-Al steels under industrial conditions," Materials Science Forum, vol. 500-501, pp. 195-202, 2005.

[27] L. Durrenberger, A. Molinari, and A. Rusinek, "Internal variable modeling of the high strain-rate behavior of metals with applications to multiphase steels," Materials Science and Engineering A, vol. 478, no. 1-2, pp. 297-304, 2008.

[28] A. Molinari and G. Ravichandran, "Constitutive modeling of high-strain-rate deformation in metals based on the evolution of an effective microstructural length," Mechanics of Materials, vol. 37, no. 7, pp. 737-752, 2005.

[29] C. Y. Gao and L. C. Zhang, "A constitutive model for dynamic plasticity of FCC metals," Materials Science and Engineering A, vol. 527, no. 13-14, pp. 3138-3143, 2010.

[30] L.-E. Lindgren, K. Domkin, and S. Hansson, "Dislocations, vacancies and solute diffusion in physical based plasticity model for AISI 316L," Mechanics of Materials, vol. 40, no. 11, pp. 907-919, 2008.

[31] J. A. Rodríguez-Martínez, M. Rodríguez-Millán, A. Rusinek, and A. Arias, "A dislocation-based constitutive description for modeling the behavior of FCC metals within wide ranges of strain rate and temperature," Mechanics of Materials, vol. 43, no. 12, pp. 901-912, 2011.
[32] S. M. Byon, S. I. Kim, and Y. Lee, "Predictions of roll force under heavy-reduction hot rolling using a large-deformation constitutive model," Proceedings of the Institution of $\mathrm{Me}$ chanical Engineers - Part B: Journal of Engineering Manufacture, vol. 218, no. 5, pp. 483-494, 2004.

[33] X. Feng, X. Wang, Q. Yang, and J. Sun, "Analysis of rate dependency on roll force calculation during hot strip rolling based on Karman equation," Advances in Mechanical Engineering, vol. 11, no. 1, Article ID 168781401882493, 2019.

[34] J. S. Wang, Z. Y. Jiang, A. K. Tieu, X. H. Liu, and G. D. Wang, "Adaptive calculation of deformation resistance model of online process control in tandem cold mill," Journal of Materials Processing Technology, vol. 162-163, pp. 585-590, 2005.

[35] Y. K. Sun, Model and Control of Hot and Cold Strip Mill, pp. 41-42, Metallurgical Industry Press, Beijing, China, 2010.

[36] F. D. Foresee and T. H. Martin, "Gauss-Newton approximation to Bayesian learning," in Proceedings of the international conference on neural networks (ICNN'97), vol. 3, pp. 1930-1935, Houston, TX, USA, June 1997.

[37] J. M. Hernández-Lobato and R. P. Adams, "Probabilistic backpropagation for scalable learning of bayesian neural networks," in Proceedings of the International Conference on Machine Learning PMLR, pp. 1861-1869, Lille, France, July 2015.

[38] L. Q. Han, Artificial Neural Networks Tutorial, pp. 69-70, Beijing University of Posts and Telecommunications Press, Beijing, China, 2006.

[39] Y. Saito, T. Enami, and T. Tanaka, "The mathematical model of hot deformation resistance with reference to microstructural changes during rolling in plate mill," Transactions of the Iron and Steel Institute of Japan, vol. 25, no. 11, pp. 1146-1155, 1985.

[40] Y. Y. He, X. Yan, and L. X. Li, "Stress state coefficient model of CSP line," Journal of Iron and Steel Research, vol. 25, no. 9, pp. 16-18, 2013. 